\title{
Three-dimensional culture and interaction of cancer cells and dendritic cells in an electrospun nano-submicron hybrid fibrous scaffold
}

This article was published in the following Dove Press journal:

International Journal of Nanomedicine

2 March 2016

Number of times this article has been viewed

\author{
Tae-Eon Kim, ${ }^{1-3, *}$ Chang \\ Gun Kim, ${ }^{1-3, *}$ Jin Soo Kim, ${ }^{4}$ \\ Songwan Jin, ${ }^{4}$ Sik Yoon, ${ }^{5}$ \\ Hae-Rahn Bae, ${ }^{6}$ Jeong-Hwa \\ Kim, ${ }^{7,8}$ Young Hun Jeong, ${ }^{7,8}$ \\ Jong-Young Kwak ${ }^{1-3}$ \\ 'Department of Pharmacology, \\ School of Medicine, ${ }^{2}$ Department of \\ Biomedical Sciences, The Graduate \\ School, Ajou University, Suwon, South \\ Korea; ${ }^{3}$ mmune Network Pioneer \\ Research Center, Ajou University \\ Medical Center, Suwon, South \\ Korea; ${ }^{4}$ Department of Mechanical \\ Engineering, Korea Polytechnic \\ University, Gyeonggi, South Korea; \\ ${ }^{5}$ Department of Anatomy, School of \\ Medicine, Pusan National University, \\ Yangsan, South Korea; ${ }^{6}$ Department \\ of Physiology, College of Medicine, \\ Dong-A University, Busan, South \\ Korea; ${ }^{7}$ School of Mechanical \\ Engineering, ${ }^{8}$ Department of \\ Mechanical Engineering, Graduate \\ School, Kyungpook National \\ University, Daegu, South Korea \\ *These authors contributed equally \\ to this work
}

Correspondence: Jong-Young Kwak Department of Pharmacology, School of Medicine, Ajou University, Worldcup-Ro 164, Suwon 443-380, South Korea

$\mathrm{Tel}+82312195064$

Fax +82312195069

Email jykwak@dau.ac.kr

Young Hun Jeong

School of Mechanical Engineering,

Kyungpook National University,

Daegu 702-70I, South Korea

Tel +82539505577

Email yhjeong@knu.ac.kr

\begin{abstract}
An artificial three-dimensional (3D) culture system that mimics the tumor microenvironment in vitro is an essential tool for investigating the cross-talk between immune and cancer cells in tumors. In this study, we developed a 3D culture system using an electrospun poly( $\varepsilon$-caprolactone) (PCL) nanofibrous scaffold (NFS). A hybrid NFS containing an uninterrupted network of nano- and submicron-scale fibers ( $400 \mathrm{~nm}$ to $2 \mu \mathrm{m}$ ) was generated by deposition onto a stainless steel mesh instead of an aluminum plate. The hybrid NFS contained multiplanar pores in a 3D structure. Surface-seeded mouse CT26 colon cancer cells and bone marrowderived dendritic cells (BM-DCs) were able to infiltrate the hybrid NFS within several hours. BM-DCs cultured on PCL nanofibers showed a baseline inactive form, and lipopolysaccharide (LPS)-activated BM-DCs showed increased expression of CD86 and major histocompatibility complex Class II. Actin and phosphorylated FAK were enriched where unstimulated and LPSstimulated BM-DCs contacted the fibers in the 3D hybrid NFS. When BM-DCs were cocultured with mitoxantrone-treated CT26 cells in a 3D hybrid NFS, BM-DCs sprouted cytoplasm to, migrated to, synapsed with, and engulfed mitoxantrone-treated CT26 cancer cells, which were similar to the naturally occurring cross-talk between these two types of cells. The 3D hybrid NFS developed here provides a 3D structure for coculture of cancer and immune cells.
\end{abstract}

Keywords: 3D cell culture, electrospinning, nanofibrous scaffold, dendritic cell, colon cancer cell

\section{Introduction}

In vivo, cells occupy a three-dimensional (3D) environment. Investigating cell functions such as adhesion, infiltration, and migration requires accurate mimicry of the in vivo microenvironment. Therefore, 3D culture techniques are garnering increasing attention in the field of cell biology, for the generation of microenvironments that mimic physiological conditions. Fabricated nanofibrous mats have previously been developed for use in tissue engineering. ${ }^{1}$ Recently, mats consisting of fabricated and patterned nanofibrous scaffolds (NFS) have been developed for cell culture. ${ }^{2}$ Ideally, NFS should mimic the ability of the extracellular matrix (ECM) to bind cells in a 3D manner. Although various types of 3D scaffolds have been developed, most only promote cell adhesion, proliferation, and migration on the surface, because they are composed of highly packed fabrics or high-surface-density fibers. ${ }^{3}$ An NFS ideal for 3D culture of cells that mimics the architecture of the natural ECM has not yet been developed because cells typically show poor infiltration of and growth inside NFS.

Randomly electrospun NFS has a similar spatial dimensionality to the fibrous component of the ECM, which consists of a nanoscale fibrous network of proteins 
and proteoglycans. For efficient 3D cell ingrowth, a sufficiently large pore size is required. Structures that combine nano- and submicron-scale fibers have been developed by random electrospinning of nanoscale fibers on microscale fibers but provide only nanobridges between microfibers. ${ }^{4}$ Dual-scale 3D scaffolds have also been developed by deposition of electrospun nanofibers between microfibrous layers, which were fabricated using direct polymer melt deposition. ${ }^{5}$ However, the pores in these scaffolds are still too small to allow cell infiltration.

The immune system can either promote or inhibit tumor progression. Tumor-infiltrating leukocytes are a heterogeneous population of immune cells. A limited number of in vitro studies have been published on the contribution of immune cells to tumor progression, due to the lack of a 3D coculture system that mimics the in vivo tumor microenvironment. For this study, dendritic cell (DC) was chosen as the immune cell model, because in peripheral tissues, DCs uptake tumor antigens, migrate to secondary lymphoid tissues, and present the processed antigens to T-cells. ${ }^{6}$ Results of an in vivo study by Tesniere et $\mathrm{al}^{7}$ suggested that after chemotherapy of tumors, including colon cancer, dying cells were engulfed by DCs before disintegration of the plasma membrane. ${ }^{7}$ Therefore, the development of a $3 \mathrm{D}$ coculturing system is essential for the in vitro investigation of the engulfment of dying cancer cells by DCs. However, to date, a method for in vitro preparation of $3 \mathrm{D}$ culture that can be used to investigate interactions between DCs and cancer cells has not been established. Moreover, cellular functions such as adhesion, spreading, migration, and activation have not been studied in DCs cocultured with cancer cells in a 3D NFS.

Nanofibrous poly( $\varepsilon$-caprolactone) (PCL) mats have been used as scaffolds to promote proliferation and differentiation of various kinds of cells because they are highly biocompatible. ${ }^{8,9}$ However, it remains unknown whether electrospun PCL fibers are unreactive with immune cells such as DCs and are thus a suitable material for immune cell culture. We developed a 3D NFS that consists of nanoscale fibers (400-800 $\mathrm{nm}$ in diameter) and submicron-scale fibers (1-2 $\mu \mathrm{m}$ in diameter), termed a hybrid NFS. Because nanoand submicron-scale fibers are dispersed within the hybrid NFS, they provide a stable $3 \mathrm{D}$ structure that promotes $3 \mathrm{D}$ adhesion, spreading, migration, and function of DCs in coculture with cancer cells.

\section{Materials and methods}

\section{Materials}

PCL $\left(M_{\mathrm{n}}=700,000-900,000\right)$, chloroform, fluorescein isothiocyanate (FITC)-labeled albumin, and lipopolysaccharide
(LPS) were purchased from Sigma-Aldrich (St Louis, MO, USA). Dulbecco's Modified Eagle's Medium, fetal bovine serum (FBS), and $0.05 \%$ trypsin-ethylenediaminetetraacetic acid were purchased from Gibco (Rockville, MD, USA). Granulocyte macrophage-colony stimulating factor, interleukin-4, carboxyfluorescein succinimidyl ester, FITC-phalloidin, and antibodies against CD11, CD86, major histocompatibility complex (MHC) Class II, calreticulin, phosphorylated focal adhesion kinase (p-FAK), and reticular fiber were obtained from R\&D Systems Inc. (Minneapolis, MN, USA). 4',6Diamidino-2-phenylindole (DAPI) and CellTracker Red CMTPX were purchased from Invitrogen (Carlsbad, CA, USA). Collagen Type I was supplied by Merck Millipore (Darmstadt, Germany). Fluorescent membrane dyes, PKH26 and PKH67, were purchased from Molecular Probes, Inc. (Eugene, OR, USA). Polydimethylsiloxane (PDMS) was purchased from Dow-Corning Korea, Inc. (Seoul, South Korea).

\section{Electrospinning and fabrication of the hybrid PCL scaffold}

The polymer for electrospinning was dissolved in $99.5 \%$ pure chloroform at a concentration of $8.8 \mathrm{wt} \%$ and stirred for 5 hours to obtain a homogeneous solution. Two types of nanofiber mats with the same dimensions were fabricated by electrospinning. The first was a conventionally electrospun mat (compact NFS), and the second contained larger pores (hybrid NFS) to encourage cell migration into the mat. Both types of mats were fabricated on a collector plate and were nearly flat. Figure 1A shows a schematic of the experimental electrospinning setup constructed for the fabrication of these mats. Four-nozzle spinnerets with $12 \mathrm{~mm}$ nozzle spacing were used. Each nozzle had an inner diameter of approximately $210 \mu \mathrm{m}(27 \mathrm{G})$ and with an average flow rate of approximately $0.125 \mathrm{~mL} / \mathrm{h}$ for PCL-chloroform solution. To produce flat nanofiber mats, the collector plates were given reciprocating 2D planar motion, at a speed of $20 \mathrm{~mm} / \mathrm{s}$ by means of a 2D linear stage (LPP-LM11\&12, DCT Corp., Suwon, South Korea), so that the nanofibers covered most of the collector plate. The nozzle-tip-to-collector distance was set at $100 \mathrm{~mm}$, with an electrical potential of approximately $11 \mathrm{kV}$ from the grounded collector plate. Two types of collector plates were used: a solid aluminum plate and a stainless steel mesh. The aluminum plate was used for fabricating conventionally electrospun nanofiber mats, and the stainless steel mesh, which aided in deposition guidance, was used for fabricating nanofiber mats with larger pores. ${ }^{10,11}$ The wire diameter and grid size of the mesh were $250 \mathrm{~mm}$ and $1.4 \mathrm{~mm}^{2} \times 1.2 \mathrm{~mm}^{2}$, respectively. The mesh was covered 
A

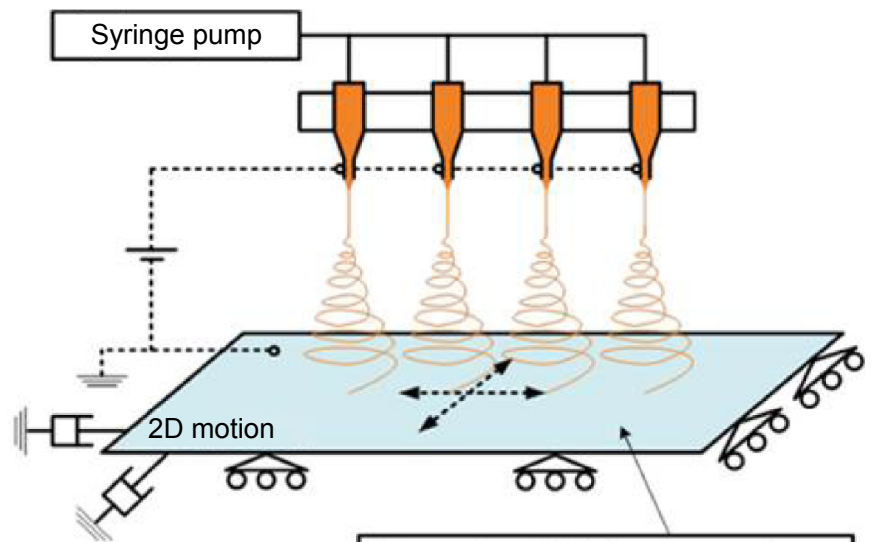

Type 1: solid aluminum plate

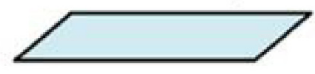

Type 2: stainless steel mesh

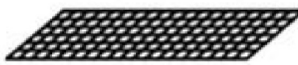

B
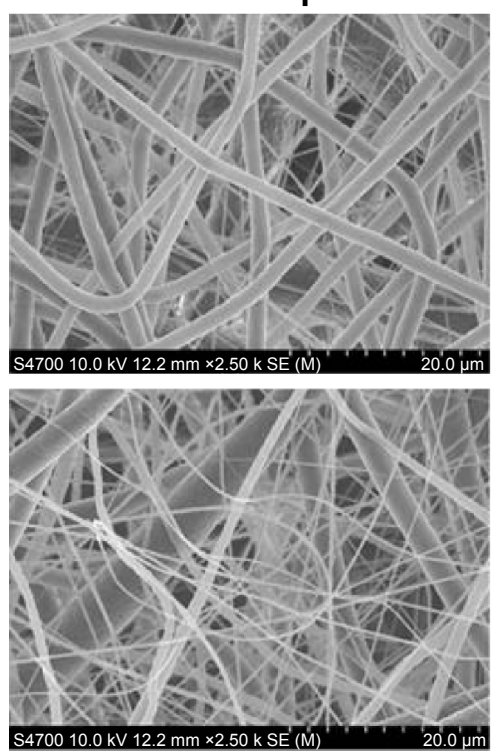

Mesh plate/glass

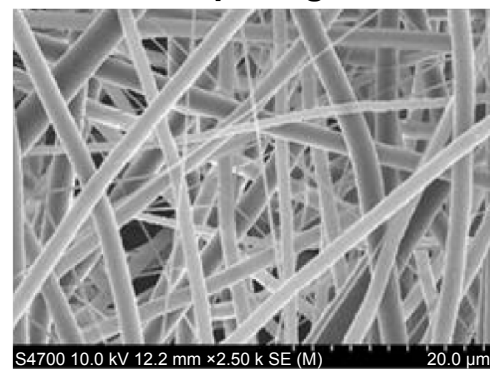

Upper

surface

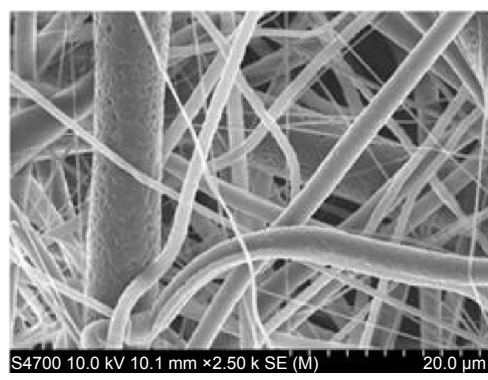

Lower surface

Figure I Production of hybrid NFS.

Notes: (A) Electrospinning setup for the production of compact and hybrid NFS. (B) Fiber morphology in compact and hybrid NFS. SEM images of compact NFS electrospun on aluminum plates and hybrid NFS electrospun on glass over stainless steel mesh.

Abbreviations: NFS, nanofibrous scaffold; SEM, scanning electron microscope; 2D, two dimensional.

with a $2 \mathrm{~mm}$ thick glass plate to weaken the electrical field strength and to make it easy to detach the nanofibers. The solid and mesh collector plates had identical dimensions of $120 \mathrm{~mm}^{2} \times 120 \mathrm{~mm}^{2}$, which corresponded to the mat dimensions. The fabrication time for each nanofiber mat was set at 5 hours, regardless of mat type.

\section{Morphological analysis of hybrid NFS}

The thicknesses of the fabricated compact and hybrid NFS were measured using an ultraprecision micrometer (369-250, Mitsutoyo Co., Kawasaki, Japan). The pores in the interfiber regions of the fabricated NFS were investigated using a mercury porosimeter (AutoPore IV 9500, Micromeritics Instrument Co., Norcross, GA, USA) with a penetrometer ( $\mathrm{s} / \mathrm{n}=14,3 \mathrm{bulb}, 0.412 \mathrm{stem}$, powder). The samples for porosimetry were cut into $30 \times 50 \mathrm{~mm}^{2}$ rectangles. The mercury filling pressure and equilibrium time were $1.23 \mathrm{psi}$ and 10 seconds, respectively. NFS morphology was observed using a field-emission scanning electron microscope (SEM) (S4700, Hitachi Co, Tokyo, Japan) and fiber diameters were measured using image analysis software (ImageJ, National Institutes of Health, Bethesda, MD, USA). 


\section{Culture of bone marrow-derived DCs and cancer cells}

Mouse bone marrow-derived DCs (BM-DCs) were obtained from mouse BM precursors, as described previously. ${ }^{12}$ Briefly, BM cells were harvested from the femurs and tibias of BALB/c mice and then plated in complete RPMI 1640 containing recombinant murine granulocyte macrophagecolony stimulating factor $(20 \mathrm{ng} / \mathrm{mL})$ plus recombinant murine interleukin-4 (20 ng/mL). On day 7 of culture, immature BM-DCs, which appeared as nonadherent proliferating aggregates, were collected and the percentage of BM-DCs was verified by flow cytometry using surface markers (CD11c and MHC Class II). LPS ( $1 \mu \mathrm{g} / \mathrm{mL})$ was added to the wells or NFS for 24 hours to induce DC activation, which was evaluated by flow cytometry. CT26 murine colon carcinoma cells, syngeneic to BALB/c mice, were grown as monolayer cultures in Dulbecco's Modified Eagle's Medium containing 10\% FBS supplemented with $100 \mathrm{IU} / \mathrm{mL}$ penicillin and $100 \mu \mathrm{g} / \mathrm{mL}$ streptomycin. Nanofibrous mats were attached to PDMS-coated slide glasses. A cell culture chamber was constructed using a plastic dam, and cells were cultured in the compartment on the mat. CT26 cancer cells, grown either in culture dish or in hybrid NFS, were treated with or without mitoxantrone for 1 hour, washed with culture media, and further cultured for 18 hours. Untreated or mitoxantrone-treated CT26 cancer cells were seeded at a density of $5 \times 10^{4}$ cells $/ \mathrm{cm}^{2}$ and cocultured with BM-DCs at a 1:5 ratio of BM-DCs to CT26 cells.

\section{Culture of cells derived from tumor tissues}

$\mathrm{BALB} / \mathrm{c}$ mice were maintained in accordance with Ajou University Hospital institutional animal care and the institute approved the study. CT26 colon cancer cells (106/mouse) in $100 \mu \mathrm{L}$ of phosphate-buffered saline (PBS) were implanted into the lower right flanks of mice. On day 14, these mice were sacrificed and tumor tissues were digested with DNase ( $1 \mu \mathrm{g} / \mathrm{mL}$, Grade II bovine pancreatic DNase) and collagenase Type II ( $1 \mathrm{mg} / \mathrm{mL})$ to generate single-cell suspensions.

\section{Laser confocal microscopy}

The morphology of cells that had infiltrated the NFS was observed using an Olympus confocal microscope FV1000 (Olympus Corporation, Tokyo, Japan; n=5). Image analysis was performed using the ImageJ software. Briefly, BM-DCs and CT26 cancer cells in the culture dish and hybrid NFS were fixed with $4 \%$ paraformaldehyde, permeabilized with $0.2 \%$ Triton-X-100, and then stained with DAPI $(1 \mu \mathrm{g} / \mathrm{mL})$,
FITC-phalloidin (1 unit/mL), and fluorescent-labeled antibodies in PBS for 30 minutes. Phenotypic activation of BM-DCs was measured by immunofluorescence microscopic analysis of CD86 and MHC Class II upregulation.

\section{Cell proliferation assay}

The viability of CT26 cells in culture dish and hybrid NFS was assessed using a cell counting kit (CCK)-8 assay, as per the manufacturer's protocol (Cell Counting Kit-8; Dojindo Molecular Technologies, Gaithersburg, MD, USA; n=3).

\section{Statistical analysis}

Results are presented as means \pm standard deviation. Student's $t$-test was used to compare the means of paired or unpaired samples. A $P$-value of $<0.05$ was considered significant.

\section{Results}

\section{Electrospun hybrid NFS}

When the thicknesses of the compact and hybrid NFS were compared, the compact NFS had an average thickness of approximately $50 \mu \mathrm{m}(50.2 \pm 2.6 \mu \mathrm{m})$, whereas the average thickness of the hybrid NFS was approximately $54 \mu \mathrm{m}(54.0 \pm 4.0 \mu \mathrm{m} ; \mathrm{n}=18)$. Compact and hybrid NFS were electrospun with equal amounts of polymer and there was no statistical difference in their mat thickness. The ultrastructure of compact and hybrid NFS was analyzed by SEM to assess fiber size, fiber uniformity, and surface topography. When the fiber morphology of the lower and upper surfaces of compact and hybrid NFS was examined, it varied depending on locality because of changes in electrical field strength (Figure 1B). The lower surface, which was closer to the collector plate, contained more fine-diameter fibers, whereas more thick fibers were deposited on the upper surface. The nanofiber content deposited on the lower region of the scaffolds spun on the mesh collector was significantly lesser than that deposited on the aluminum plate. In the upper region, the hybrid and compact NFS showed similar fiber morphology. When nanofibers were collected on a stainless steel mesh plate, their average diameter increased to $2-3 \mu \mathrm{m}$. Fiber diameter distributions, given in count rate per area of compact or hybrid NFS, are shown in Figure 2A. In all cases, the peak frequency of fiber diameter lay between 200 and $400 \mathrm{~nm}$, and a significant number of microfibers with a diameter of 1-2 $\mu \mathrm{m}$ were deposited. Comparing the fiber diameter distribution, hybrid NFS contained approximately twofold more microfibers with a diameter of 1-2 $\mu \mathrm{m}$ than compact NFS in the lower region, and approximately $70 \%$ 

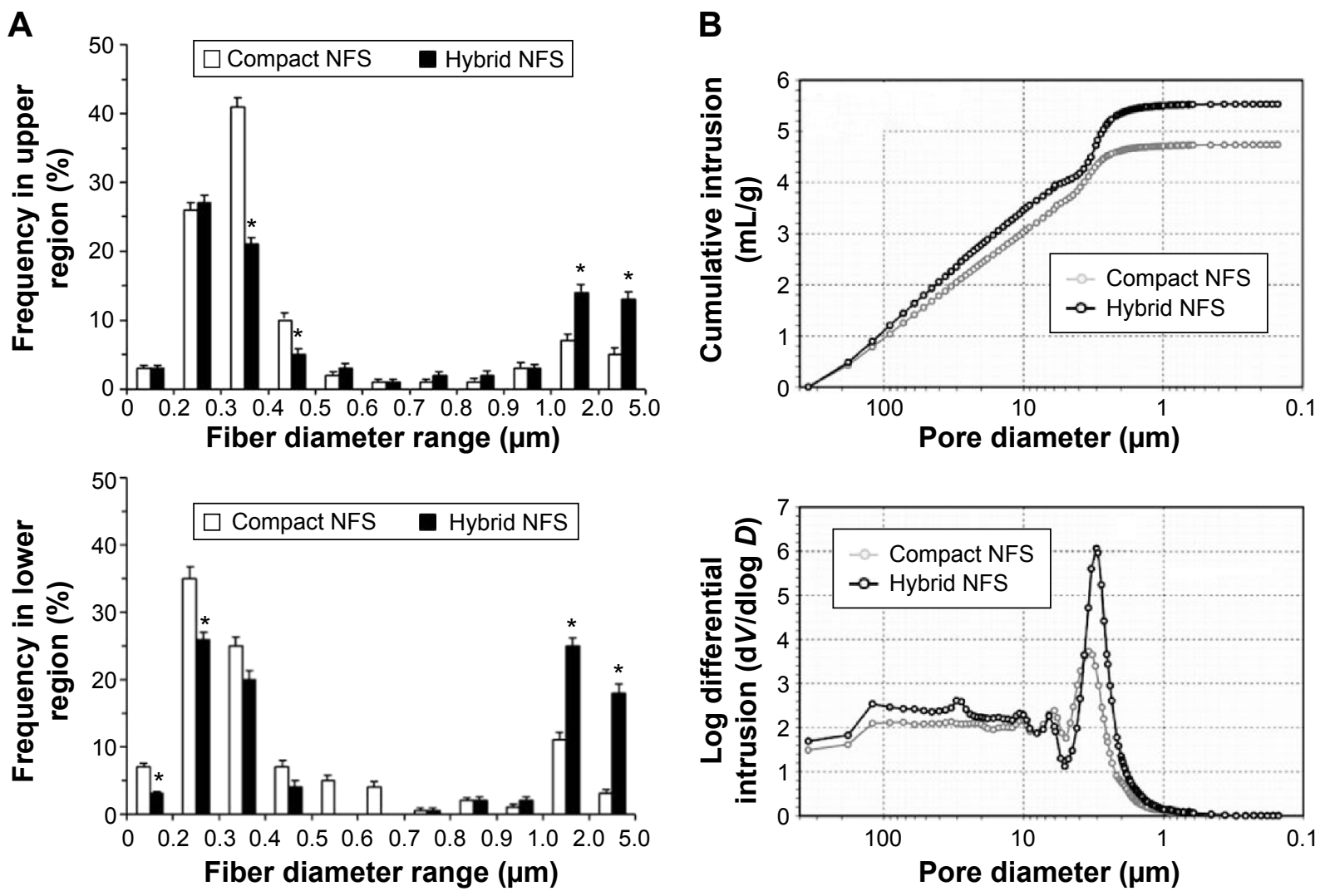

Figure 2 Fiber diameter and pore size distribution of compact and hybrid NFS.

Notes: (A) Fiber diameter frequency in top (upper panel) and bottom (lower panel) layers of the fibrous scaffolds measured by analysis of SEM images of NFS. Data are shown as mean $\pm S D(n=5)$. (B) Cumulative mercury intrusion curve (upper panel) and logarithmic differential mercury intrusion curve (lower panel) measured using mercury porosimetry. Data are representative of three independent experiments. Asterisks $\left(^{*}\right)$ denote significant differences compared to compact NFS $(P<0.05)$.

Abbreviations: NFS, nanofibrous scaffold; SEM, scanning electron microscope; SD, standard deviation.

more in the upper region. The lower and upper regions of hybrid NFS contained approximately two- and fourfold more microfibers $>2 \mu \mathrm{m}$ in diameter. Hybrid NFS can be inferred to contain larger pores than compact NFS because, in general, microfibers introduce larger pores than nanofibers.

When porosity data for compact and hybrid NFS measured by mercury porosimetry, hybrid NFS had 17\% higher total mercury intrusion volume than compact NFS (Table 1). Hybrid NFS had $12 \%$ higher porosity than compact NFS, as expected. Pore size distribution was also determined from the mercury intrusion data. The cumulative intrusion curve according to pore diameter (equivalently, pore size) is shown in Figure 2B. The total intrusion volume in hybrid NFS was greater than that in compact NFS, in concordance with the data presented in Table 1. Hybrid NFS also showed greater intrusion into pores with a diameter of approximately 10-100 and $2-3 \mu \mathrm{m}$. The logarithmic differential mercury intrusion, which shows the intrusion volume into pores of each specific diameter, demonstrates the contribution of each pore size to intrusion. Hybrid NFS showed a greater amount of mercury intrusion at a pressure range corresponding to the equivalent diameter range (2-4 $\mu \mathrm{m})$ than compact NFS, and it also contained more pores with a diameter of 10-100 $\mu \mathrm{m}$ than compact NFS. Thus, the hybrid NFS contained pores larger than the typical size of a cell (approximately $10 \mu \mathrm{m}$ ), and it therefore provided a better environment for cell infiltration than compact NFS.

\section{Cancer cells and BM-DCs deeply infiltrate hybrid NFS}

Because the fibrous scaffold was subjected to floating or deforming when used in culture medium, it was fixed on a slide glass using PDMS before immersion. CT26 colon

Table I Porosity information of compact and hybrid NFS measured using mercury porosimetry $(n=5)$

\begin{tabular}{lll}
\hline Parameters & Compact NFS & Hybrid NFS \\
\hline Total intrusion volume $(\mathrm{mL} / \mathrm{g})$ & $4.74 \pm 0.07$ & $5.53 \pm 0.14^{\mathrm{a}}$ \\
Total pore area $\left(\mathrm{m}^{2} / \mathrm{g}\right)$ & $2.34 \pm 0.02$ & $3.04 \pm 0.04^{\mathrm{a}}$ \\
Porosity $(\%)$ & $64.5 \mathrm{I} \pm 0.57$ & $76.53 \pm 0.09^{\mathrm{a}}$ \\
\hline
\end{tabular}

Notes: ${ }^{\mathrm{a}}$ This denotes significant differences compared to compact NFS $(P<0.05)$.

Data are shown as mean \pm SD.

Abbreviation: NFS, nanofibrous scaffold. 
cancer cells and BM-DCs became attached to the surface of compact and hybrid NFS after seeding on the top layer of the nanofibrous mats. The spreading and infiltration potentials of cancer cells and BM-DCs in hybrid NFS were evaluated 4 hours after seeding the cells on the mats. Representative SEM micrographs of cancer cells and BM-DCs cultured in compact and hybrid NFS $(n=5)$ are shown in Figure 3A. CT26 cells grown on compact NFS grew in flat sheets on the surface of randomly oriented nanofiber matrices but did not infiltrate the scaffold because cancer cells are much larger than the membrane pores. In contrast, CT26 cells grown on hybrid NFS adhered to nanofibers inside the scaffold. BM-DCs seeded on compact NFS showed dendritic projections on the surface of and inside the matrices. Therefore, it appears that nanoscale fibers provide contact guidance for cell spreading inside NFS, whereas submicron-scale fibers permit cell infiltration by generating larger pores. Focus stacking analysis of confocal microscopy images captured at $1.8 \mu \mathrm{m}$ intervals showed that fluorescent-labeled CT26 cells infiltrated the scaffold from the upper surface to a depth of $50 \mu \mathrm{m}$ (Figure 3B). In compact and hybrid NFS, CT26 cells (approximately $10 \mu \mathrm{m}$ ) were present at depths of $15 \pm 4$ and $29 \pm 6 \mu \mathrm{m}$, respectively $(n=5$, $P<0.05)$. Moreover, stacked confocal images showed that cells were more homogeneously distributed inside hybrid NFS than inside compact NFS. Therefore, we used hybrid NFS for 3D culture of cancer cells and BM-DCs. Hereafter, we will refer to conventional in vitro $2 \mathrm{D}$ cell culture in culture dish and 3D cell culture in hybrid NFS as 2D culture dish and 3D hybrid NFS, respectively.

\section{Culture and activation of BM-DCs in 3D hybrid NFS}

In this study, PCL was used to fabricate scaffolds. Spontaneous DC activation in scaffolds can cause problems when
A

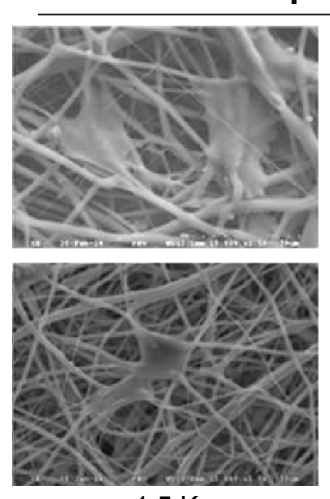

$1.5 \mathrm{~K}$
Compact NFS
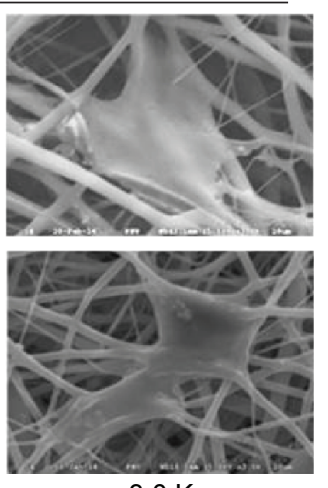

$3.0 \mathrm{~K}$
Hybrid NFS

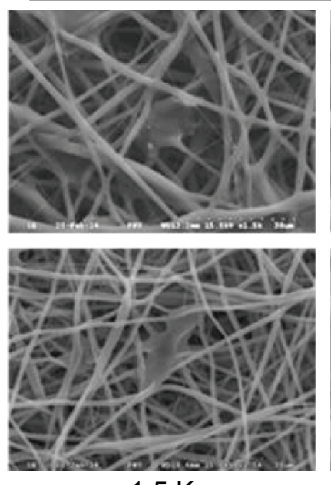

$1.5 \mathrm{~K}$

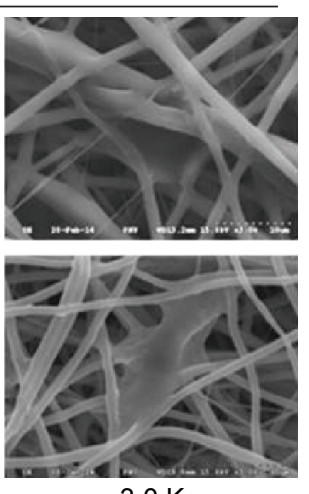

$3.0 \mathrm{~K}$
CT26 cells

BM-DCs

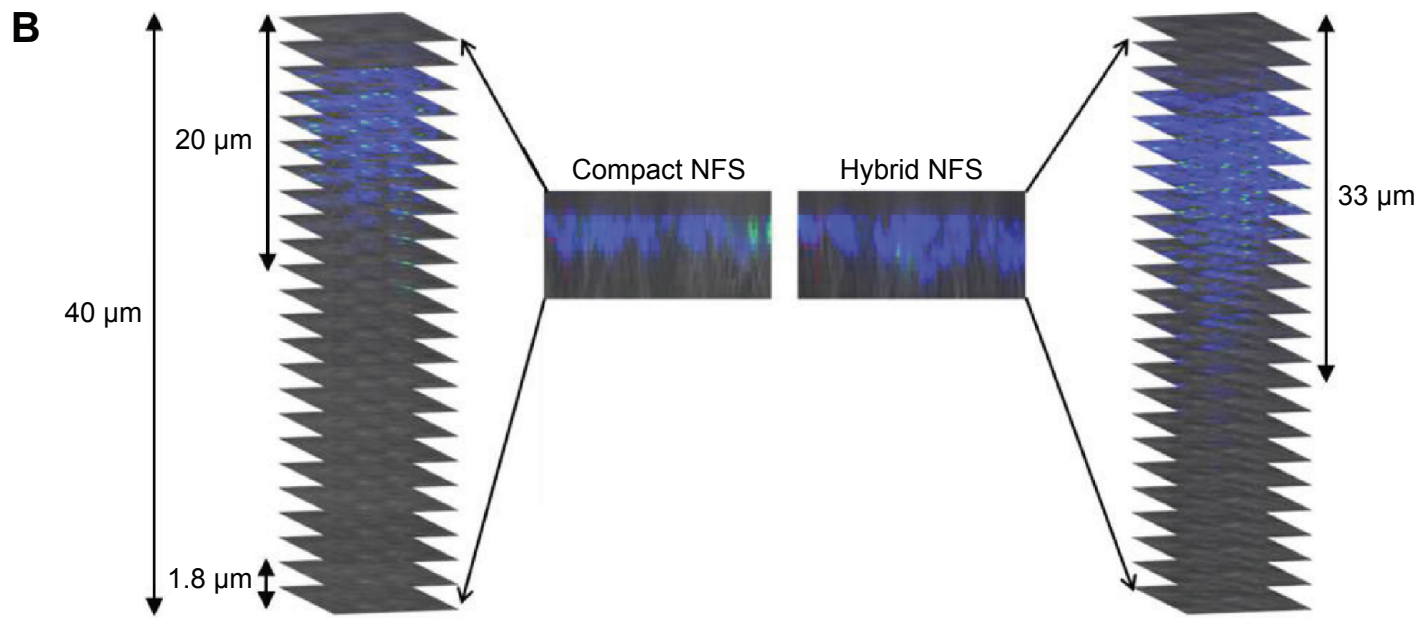

Figure 3 Infiltration of CT26 colon cancer cells and BM-DCs inside 3D hybrid NFS.

Notes: (A) SEM images of cancer cells and BM-DCs 4 hours after seeding on compact and hybrid NFS. (B) Focus stacking images of CT26 cancer cells in compact and hybrid NFS. Data are representative of three independent experiments.

Abbreviations: NFS, nanofibrous scaffold; BM-DCs, bone marrow-derived dendritic cells; SEM, scanning electron microscope. 
investigating the responses of DCs to various stimuli. Thus, we tested whether the adhesion of BM-DCs to PCL nanofibers affected their activation status. Inactive DCs express low levels of DC activation markers, such as MHC Class II and CD86, on the cell surface. ${ }^{13}$ As shown in Figure 4A, the CD86 expression level of PKH67-labeled BM-DCs was unchanged 24 hours after culture in 3D hybrid NFS, as well as in 2D culture dish, indicating that electrospun PCL nanofibers are nonreactive with DCs. Next, we investigated whether BM-DCs cultured in 3D hybrid NFS could be activated by LPS stimulation. LPS treatment for 24 hours significantly increased expression of CD86 and MHC Class II in BM-DCs cultured in 3D hybrid NFS, as well as in $2 \mathrm{D}$ culture dish. Flow cytometry analysis of LPS-stimulated DCs showed that expression levels of CD86 were similar in both conditions (data not shown). Figure 4B shows the morphological changes in unstimulated and LPS-stimulated BM-DCs in 2D culture dish and 3D hybrid NFS. To obtain cytoskeleton images, the actin in BM-DCs was stained with FITC-phalloidin. Unstimulated BM-DCs seeded in 2D culture dish were flattened, and showed actin expression on the surface attached to the dish. BM-DCs in 3D hybrid NFS were larger, and showed expression of actin along the nanofibers. LPS-stimulated BM-DCs in 2D culture dish showed more pronounced cytoplasmic projections than unstimulated cells, as well as increased expression of actin and MHC Class II in the dendrites attached to the dish surface, with similar distribution of their expression. In comparison, LPS-stimulated BM-DCs in 3D hybrid NFS showed pronounced expression of actin along nanofibers, as well as increased expression of MHC Class II throughout the cell body, including dendrites as well as nanofiber attachment sites. Thus, the conditions for culture of BM-DCs in 3D hybrid NFS differ greatly from the conditions in $2 \mathrm{D}$ culture dish, and seem to mimic in vivo conditions.

\section{Adhesion and dendrite formation of BM-DCs in 3D hybrid NFS}

We further investigated the relationship between the cytoskeletal characteristics of cultured BM-DCs and NFS topography by simultaneous observation of fluorescence images of BM-DC cytoskeletons and reflected images of nanofibers. The confocal microscopic analysis showed that actin was primarily expressed at adhesion sites of unstimulated BM-DCs and the spreading edges of LPS-stimulated BM-DCs in 2D culture dish, respectively (Figure 4C). In comparison, actin staining of unstimulated BM-DCs in 3D hybrid NFS showed the structure of the cytoskeleton where it adhered along the fibers. Moreover, although LPS-stimulated BM-DCs in 3D hybrid NFS displayed irregular cell shapes and fine processes at their edges, actin was mainly detected as discrete puncta at sites of cell adhesion to the fibers. Dynamic assembly of the focal adhesion complex has been shown to be essential for cell spreading, and FAK is known to serve as an activation marker for focal adhesions. ${ }^{14,15}$ Thus, we analyzed focal adhesion of BM-DCs cultured in 2D culture dish and 3D hybrid NFS by measuring FAK phosphorylation. Phosphorylated FAK was observed primarily on the surface of unstimulated BM-DCs where they adhered to nanofibers in 3D hybrid NFS, whereas it was primarily detected at the edges of cells in 2D culture dish. LPS stimulation induced disorganization and redistribution of phosphorylated FAK across the entire basal surface in BM-DCs in 2D culture dish. In comparison, LPS stimulation induced high enrichment of phosphorylated FAK only on the surface of cells adhered to nanofibers in 3D hybrid NFS. Moreover, LPS-activated BM-DCs in 3D hybrid NFS showed increased 3D-like dendritic projections with $\mathrm{MHC}$-II expression, whereas low levels of FAK phosphorylation were detected in dendritic projections.

\section{Coculture of BM-DCs and cancer cells in 3D hybrid NFS}

Coculture of different cell types is mainly conducted under 2D conditions, although different cell types interact under 3D conditions in tissues. We investigated whether cellular interactions between DCs and cancer cells in 3D hybrid NFS were similar to their interactions in vivo. Cocultured BM-DCs and CT2 6 cancer cells in 2D culture dish tended to form clusters, but aggregation of cocultured cells was not observed in 3D hybrid NFS (Figure 5A). Next, a preapoptotic or damaged state was induced in CT26 cancer cells by treatment with mitoxantrone, an immunogenic anticancer drug. CT26 cells were treated with mitoxantrone for 1 hour, washed, and then cultured for a further 18 hours in 2D culture dish and 3D hybrid NFS. Calreticulin is known to be expressed on the surface of cells undergoing cell death and acts as a phagocytic signal for DCs. ${ }^{16}$ Mitoxantrone-treated cells were viable 18 hours posttreatment but expressed calreticulin on their surfaces. BM-DCs were added to mitoxantrone-treated cancer cells cultured in 2D culture dish and 3D hybrid NFS and cocultured for 1 hour. BM-DCs spread to calreticulinexpressing cancer cells in 3D hybrid NFS, whereas BM-DCs interacted randomly with several calreticulin-expressing cancer cells in 2D culture dish. More specifically, as shown in Figure 5B, BM-DCs in 3D hybrid NFS extended dendrites 
A

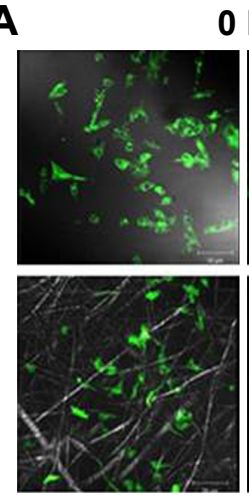

B
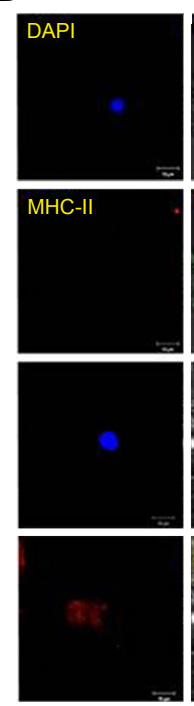

C
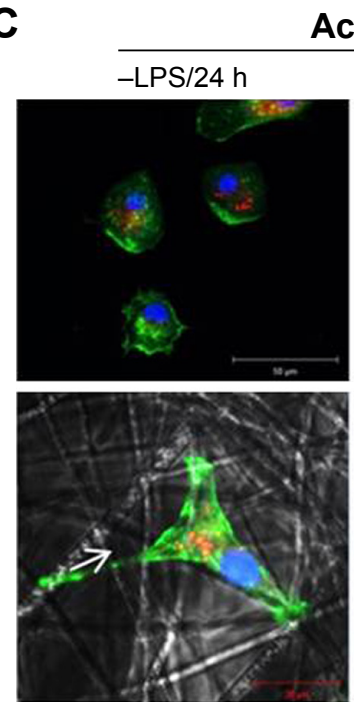

Phalloidin-FITC/MHC II-PE/DAPI (×400)

-LPS/24 h
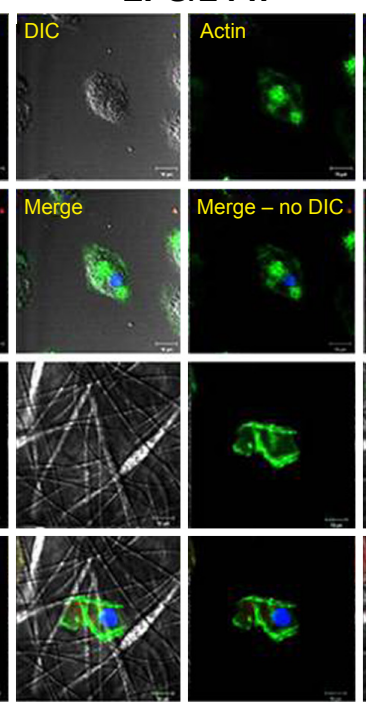

Actin
-LPS/24 h
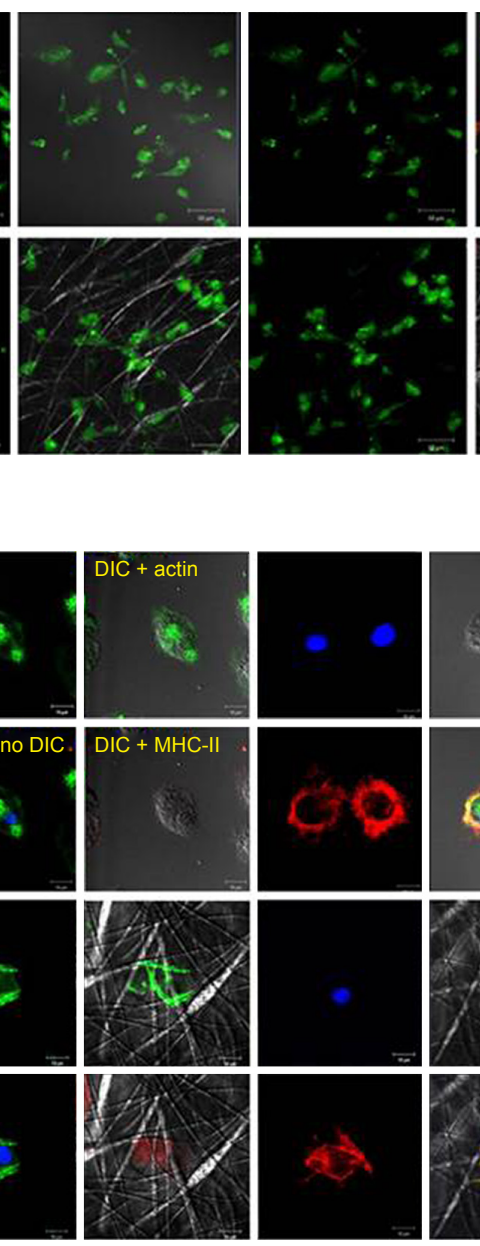

+LPS/24 h
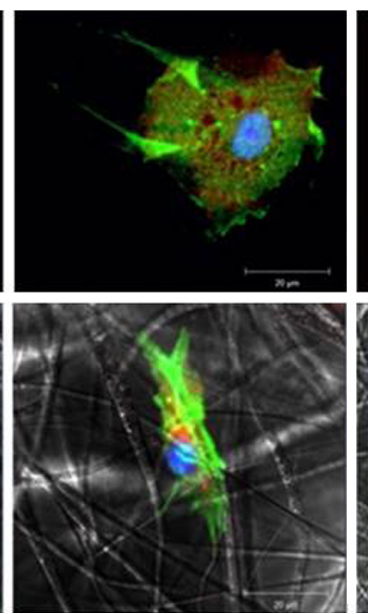

.

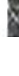

+LPS/24 h

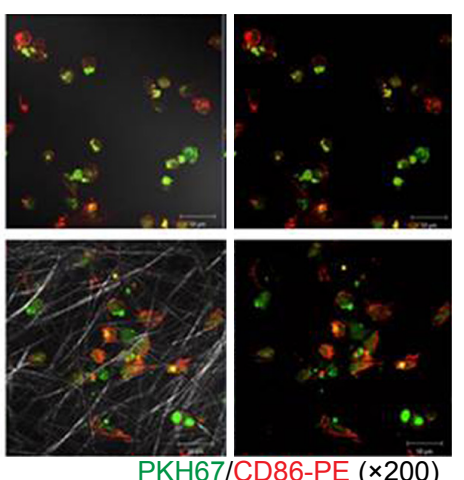

PKH67/CD86-PE (×200)

+LPS/24 h
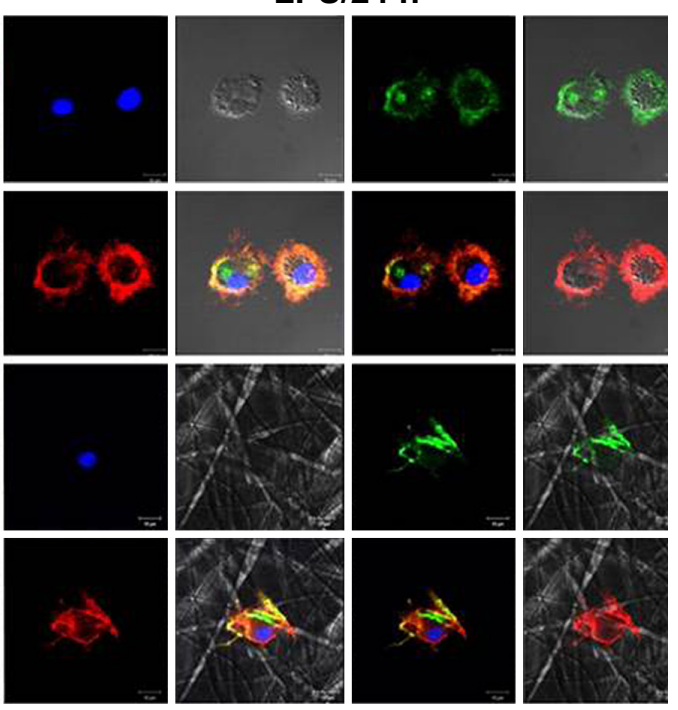

음 $\frac{\text { ㅁ }}{\frac{1}{c}}$

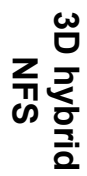

을 올

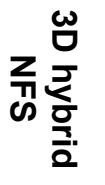

Phalloidin-FITC/MHC II-PE/DAPI (×400)
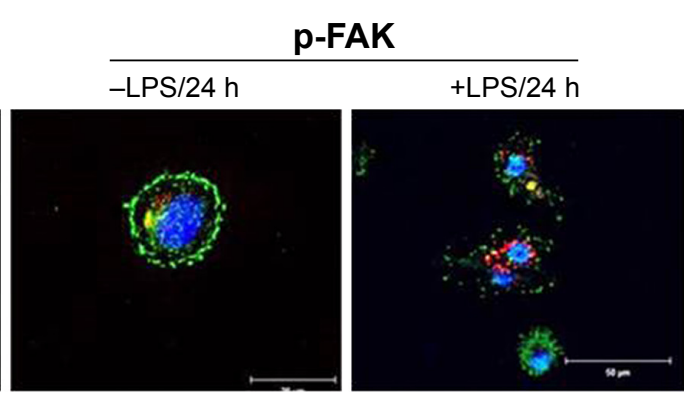

$\frac{\text { ㅁ․ }}{\text { 을 }}$
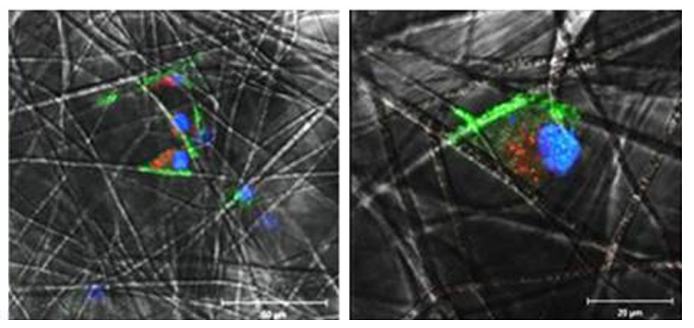

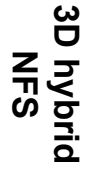

Figure 4 Activation status of untreated and LPS-activated BM-DCs in 2D culture dish and 3D hybrid NFS.

Notes: (A) Expression levels of CD86 on the surface of PKH67-labeled BM-DCs cultured with or without LPS for 24 hours in 2D culture dish and 3D hybrid NFS. (B) Confocal images of untreated and LPS-activated BM-DCs in 2D culture dish and 3D hybrid NFS using FITC-conjugated phalloidin and PE-conjugated antibody against MHC Class II. (C) Patterns of actin and phosphorylated FAK distribution in activated BM-DCs in 2D culture dish and 3D hybrid NFS. Data are representative of three independent experiments.

Abbreviations: NFS, nanofibrous scaffold; BM-DCs, bone marrow-derived dendritic cells; LPS, lipopolysaccharide; MHC, major histocompatibility complex; FAK, focal adhesion kinase; SEM, scanning electron microscope; 2D, two dimensional; 3D, three dimensional; FITC, fluorescein isothiocyanate; PE, phycoerythrin. 
A
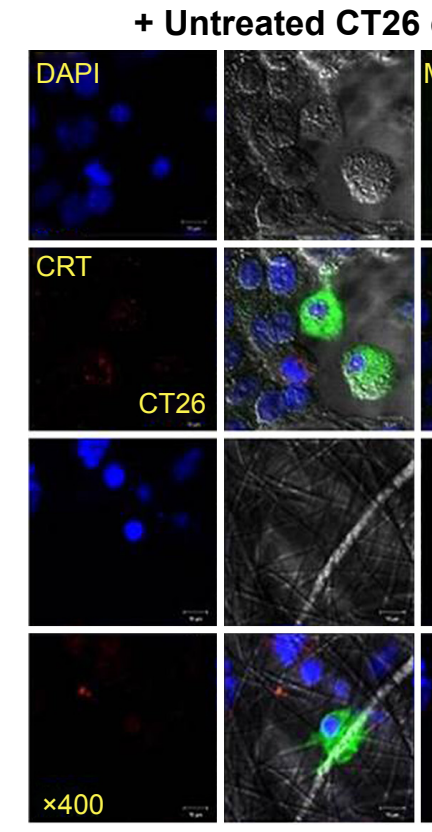
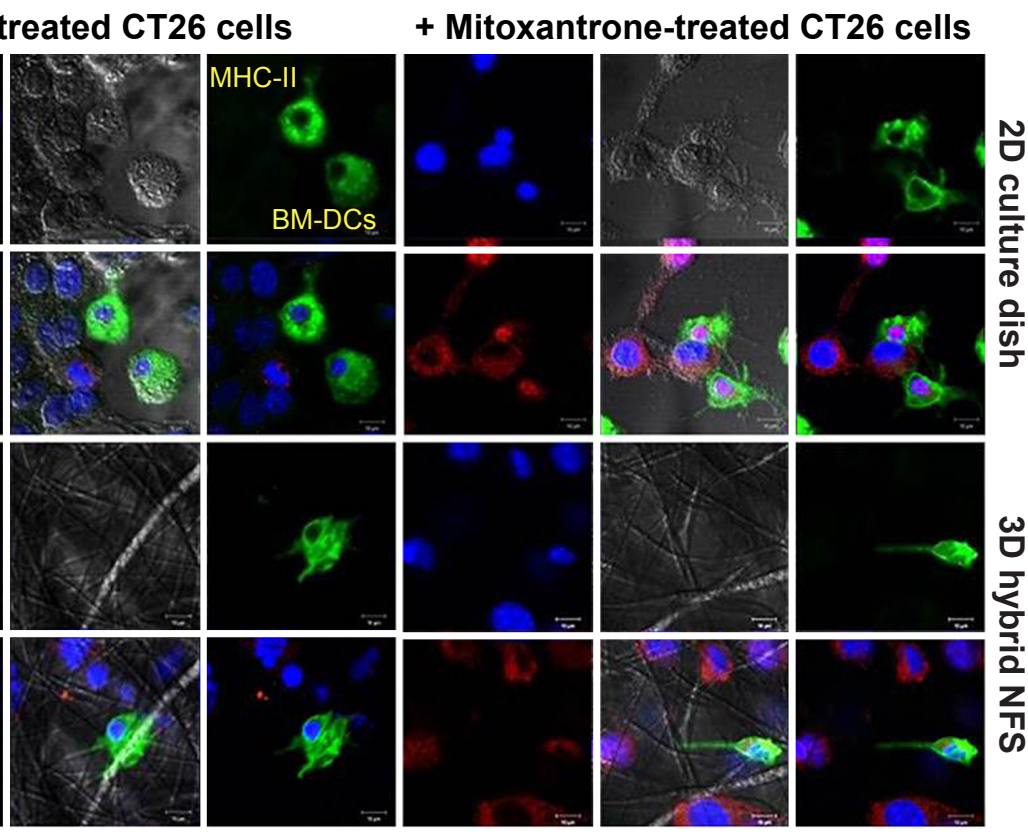

\section{B + Untreated CT26 cells}

+ Mitoxantrone-treated CT26 cells
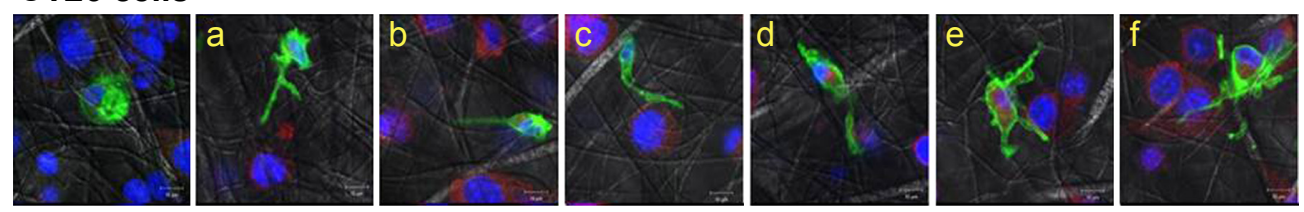

Figure 5 Coculture of BM-DCs and CT26 colon cancer cells in 2D culture dish and 3D hybrid NFS.

Notes: (A) Distribution of BM-DCs and untreated or mitoxantrone-treated CT26 cells in 2D culture dish and 3D hybrid NFS. (B) Interaction of BM-DCs with mitoxantrone-treated colon cancer cells in 3D hybrid NFS. Parts a and b, extension of BM-DC to CT26 cell; c and d, adhesion of BM-DC to CT26 cell; e and f, engulfment of CT26 cell by BM-DC. Abbreviations: NFS, nanofibrous scaffold; BM-DCs, bone marrow-derived dendritic cells; 2D, two dimensional; 3D, three dimensional.

in one direction (Figure 5B-a, b), migrated, spread, and adhered to mitoxantrone-treated cancer cells (Figure 5B-c, d). Finally, BM-DCs engulfed mitoxantrone-treated cancer cells (Figure 5B-e, f). Time-lapse microscopy clearly demonstrated that CellTracker red CMTPX-labeled BM-DCs moved along nanofibers to surround green-fluorescent-protein-expressing cancer cells (Video S1), whereas BM-DCs in 2D culture dish extended several dendrites to contact cancer cells (Video S2). These results suggest that 3D culture in hybrid NFS makes it possible to measure the acquisition of tumor antigens from cancer cells by DCs.

\section{Culture of tumor tissue-derived cells in 3D hybrid NFS}

Tumors consist of cancer cells and stromal cells such as cancer-associated fibroblasts and immune cells. ${ }^{17}$ Immunofluorescence staining of tumor sections, which were prepared from tumor-bearing mice 2 weeks after implantation of CT26 cancer cells in BALB/c mice, showed infiltration of reticular fibers and $\mathrm{CD}_{11 \mathrm{C}^{+}}$cells in tumor lesions (Figure 6A).
Total tumor-tissue-derived cells, including tumor-associated stromal and cancer cells, were cultured in 2D culture dish and 3D hybrid NFS. On day 5 of culture, cancer cells and CD45 leukocytes formed multicellular aggregates in 3D hybrid NFS, whereas a typical monolayer of cancer cells alone was observed in 2D culture dish (Figure 6B). Similar cellular aggregates and survival of $\mathrm{CD} 45^{+}$leukocytes were observed in 3D hybrid NFS but not in 2D culture dish when total cells of tumor tissues isolated from B16 melanoma-bearing C57BL/6 mice were cultured for 5 days (data not shown). Higher proliferation of cancer cells than stromal cells in 2D culture condition may affect cellular aggregates. Thus, we compared proliferation rates of cancer cells in 2D culture dish and $3 \mathrm{D}$ hybrid NFS. CT26 cells were cultured in the presence of $10 \%$ FBS in 2D culture dish and 3D hybrid NFS. A CCK-8 assay was performed to determine cell viability. As shown in Figure 7, the number of viable CT26 cells cultured in 3D hybrid NFS increased in a time-dependent manner, but was lower than that in 2D culture dish, when proliferation rates were compared from day 1 to day 3 after culture. It was 
A

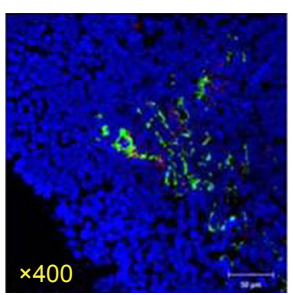

B

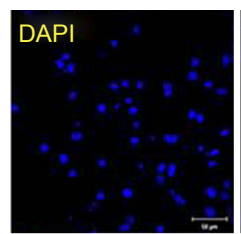

CD45
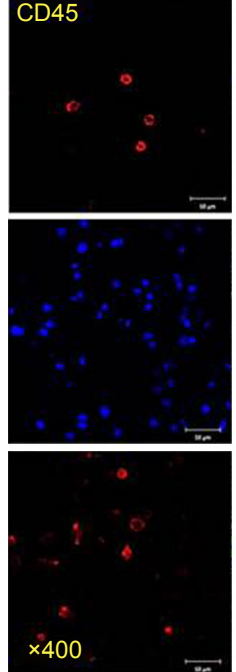

Day 1
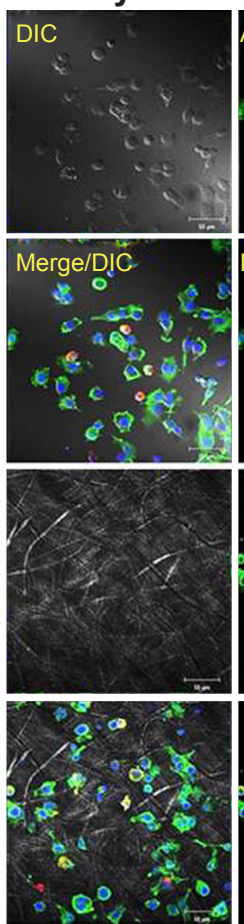
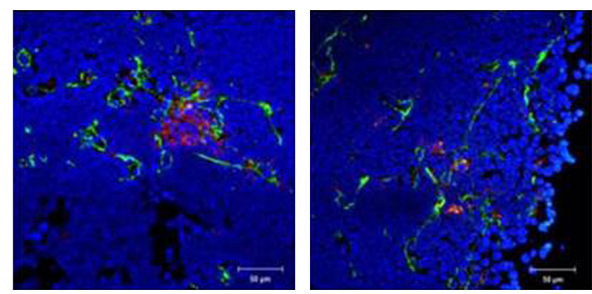

Day 5
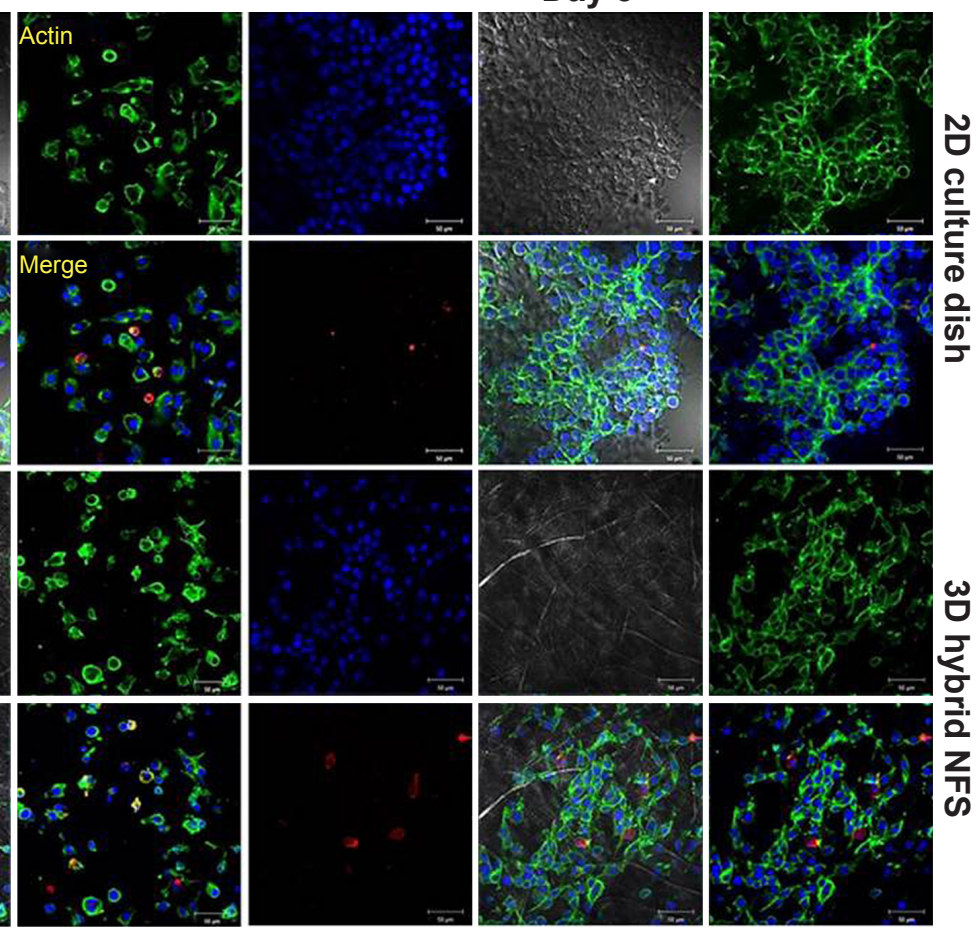

Figure 6 In vitro culture of total cells isolated from colon cancer tissue in 2D culture dish and 3D hybrid NFS

Notes: (A) Confocal analysis of tumor tissue from colon cancer-bearing mice using FITC-conjugated anti-reticular fiber antibody, PE-conjugated anti-CDI Ic antibody, and DAPI. (B) Culture of total cells isolated from tumor tissue of colon cancer-bearing mice in 2D culture dish and 3D hybrid NFS for the indicated times. Data are representative of three independent experiments.

Abbreviations: NFS, nanofibrous scaffold; DAPI, 4',6-diamino-2-phenylindole; 2D, two dimensional; 3D, three dimensional; FITC, fluorescein isothiocyanate; DIC, differential interference contrast; PE, phycoerythrin.

also demonstrated that opposite to $2 \mathrm{D}$ monolayer culture, the proliferation rate of TC-71 human Ewing sarcoma cells within the 3D PCL scaffolds was considerably lower and more similar to that of cells grown in vivo as xenografts. ${ }^{18}$ Taken together, these data show that 3D hybrid NFS can be used for in vitro modeling of tumor tissue, including cancer and stromal cells.

\section{Culture of cancer cells in collagen-coated 3D hybrid NFS}

Physiological properties of cancer cells, such as cell growth, may alter owing to the difference between PCL and collagen fibers. Therefore, collagen was electrospun into fibrous scaffolds, but the structural integrity of such scaffolds could not be retained in the aqueous environment. ${ }^{19}$ This problem was avoided by addition of collagen gel to coat PCL nanofibers. ${ }^{20}$ As shown in Figure 8A, when the solution of neutralized collagen Type I and FITC-labeled albumin was added on

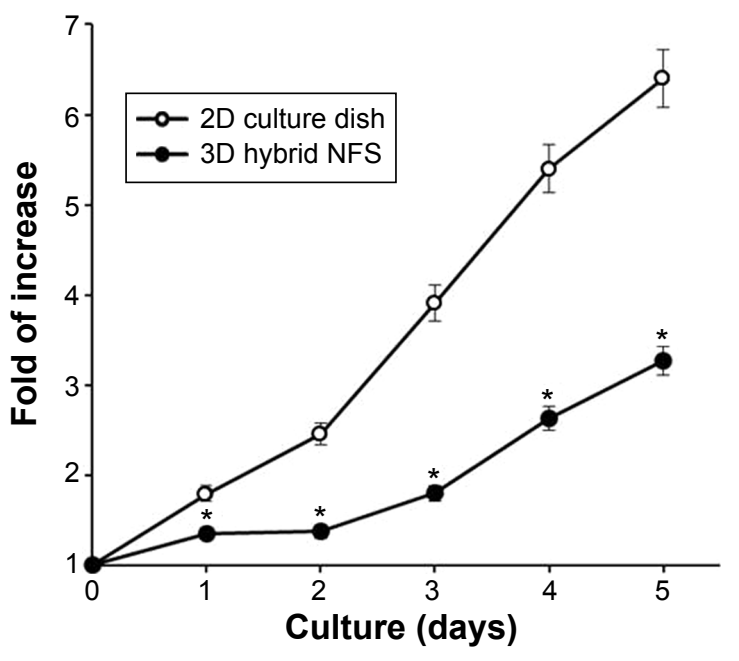

Figure 7 Proliferation of CT26 colon cancer cells in 2D culture dish and 3D hybrid NFS. Notes: CCK-8 assay of CT26 colon cancer cells cultured in 2D culture dish and $3 D$ NFS to determine viability. Data are shown as mean \pm SD $(n=3)$. Asterisks $\left(^{*}\right)$ denote significant differences compared to $2 \mathrm{D}$ culture dish $(P<0.05)$.

Abbreviations: NFS, nanofibrous scaffold; CCK, cell counting kit; 2D, two dimensional; 3D, three dimensional; SD, standard deviation. 
A

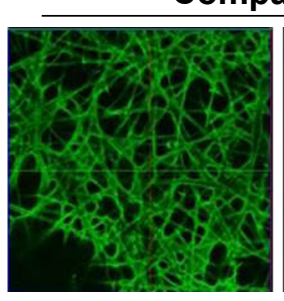

B

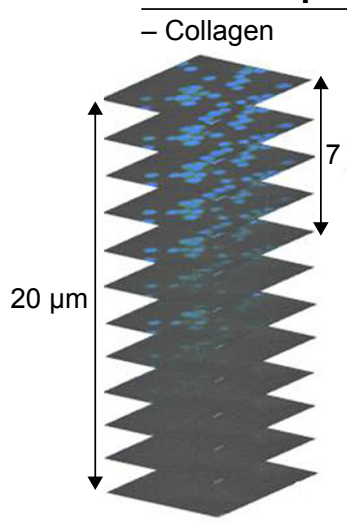

Compact NFS

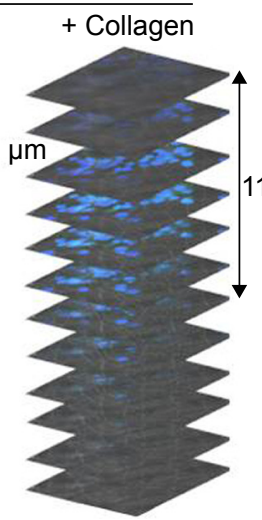

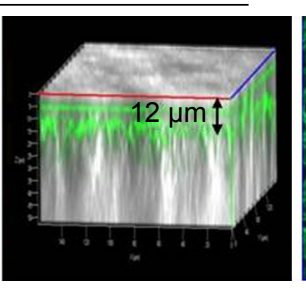

Hybrid NFS

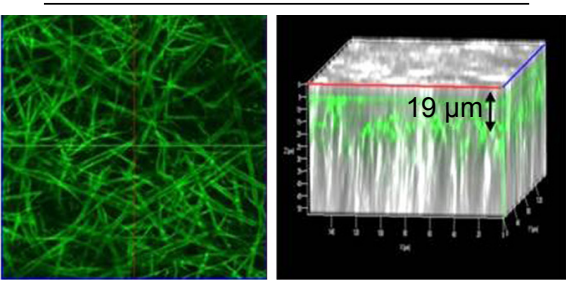

Hybrid NFS

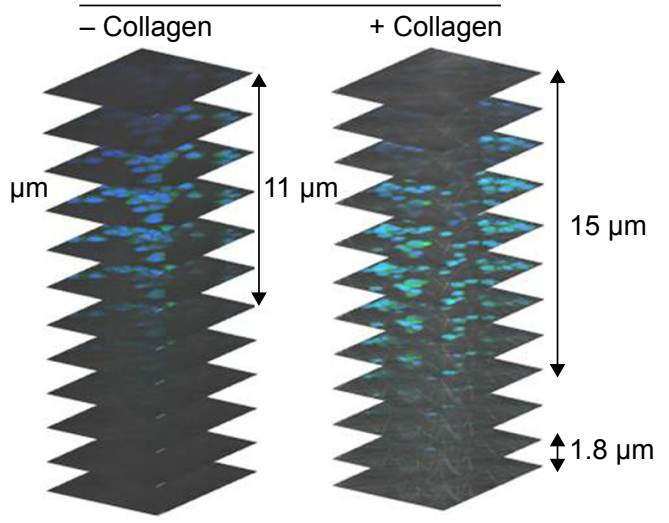

Figure 8 Collagen coating of NFS and culturing of CT26 cells with collagen solution in the compact and hybrid NFS.

Notes: (A) Confocal microscopy images of the collagen-coated NFS after addition of FITC-labeled albumin $(0.1 \mathrm{mg} / \mathrm{mL})$ and neutralized collagen Type I solution ( $0.3 \mathrm{mg} / \mathrm{mL})$. (B) Infiltration of CT26 cancer cells within the compact and hybrid NFS after seeding with neutralized collagen solution (0.3 $\mathrm{mg} / \mathrm{mL})$ on the surface of the scaffold and incubating for 4 hours. Data are representative of three independent experiments.

Abbreviations: NFS, nanofibrous scaffold; FITC, fluorescein isothiocyanate.

the surface of the compact and hybrid NFS and subsequently incubated for $4 \mathrm{~h}$ at $37^{\circ} \mathrm{C}$, nanofibers were stained with the fluorescing agent. The results of confocal microscopy indicated that fluorescent staining of nanofibers was deeper in the hybrid NFS than in the compact NFS, implying deeper diffusion of the collagen gel within the hybrid NFS. Afterward, a mixture of collagen Type I and CT26 cells was seeded on the surfaces of the compact and hybrid NFS. The addition of collagen induced deep infiltration of CT26 cancer cells into the compact and hybrid NFS after 4 hours of cell culturing (Figure 8B). Moreover, the presence of collagen provided deeper infiltration of cancer cells into the hybrid NFS than in the compact NFS. These results indicated that the presence of collagen would improve migration and adhesion of cells deeper inside the NFS and increase the biocompatibility of the scaffolds.

\section{Discussion}

In this study, we developed a 3D hybrid NFS that contains randomly oriented nanofibers of various dimensions, and which can be used as an in vitro 3D biomimetic structure. Electrospun nano- and submicron-scale fibers are intertwined in this hybrid NFS, whereas compact NFS contains only conventional uniform nanoscale fibers. The broad range of fiber diameters from nano- to submicron-scale, generated in fabrics electrospun on stainless steel mesh, produced differences in fiber density, mean pore diameter, and pore distribution, allowing cells seeded on the fiber mats to deeply infiltrate the scaffold. Our study showed that the infiltration of cancer cells and BM-DCs was dependent on an optimal mixture of nanofibers with diameters ranging from $400 \mathrm{~nm}$ to $10 \mu \mathrm{m}$. In comparison, pore size in compact NFS, composed of similar fibers ranging in diameter from $400 \mathrm{~nm}$ to $1 \mu \mathrm{m}$, appeared to be too small to allow cells to infiltrate the scaffold. It has been suggested that a macroporous polymeric scaffold, generated by altering the average pore diameter from less than a cell diameter to several times larger, allowed tracking of cellular migration within the scaffold. ${ }^{21}$ Electrospun NFS composed of fibers $>1 \mu \mathrm{m}$ in diameter permits cell infiltration, but that composed of fibers $<1 \mu \mathrm{m}$ does not. ${ }^{22,23}$ However, inside a $3 \mathrm{D}$ scaffold, cells can adhere to fibers with diameters smaller than their own. Therefore, it appears that ECM fibers control cell migration and adhesion through differences in fiber density and diameter. ${ }^{24}$ Taken together, the nano- and submicron-scale fibers in 3D hybrid NFS provide cellular adhesion sites and space for cell infiltration, respectively. On the other hand, since the cell growth and status could be affected by the wire density of NFS, the cell growing status in soft materials relative to that in dense wires should be estimated in the future studies. 
Many strategies applicable to electrospinning or postelectrospinning procedures have been proposed to enlarge the pore size of electrospun scaffolds, including combining nanofibers and microfibers into an NFS. ${ }^{25}$ A multilayered structure can be fabricated by sequentially alternating electrospinning of nanofibers and microfibers, or by mixing of nanofibers and microfibers electrospun from different syringes. ${ }^{26,27}$ However, these techniques produce small pores between the nanofiber and microfiber layers, which prevent cellular infiltration in the scaffold. The 3D hybrid NFS developed here contains larger pores to overcome this problem. 3D hybrid NFS enabled eventual adhesion, spreading, and migration of top-seeded cancer cells and BM-DCs on the surface of the mat, providing cellular anchors along nanofibers deep inside the scaffold rather than on the outermost surface alone.

In $3 \mathrm{D}$ culture systems, the scaffold promotes both constructive and functional remodeling of normal and tumor tissues, as the scaffold topography affects cellular behavior. Therefore, a designed NFS should maintain cellular functions, including proliferation, differentiation, spreading, migration, and activation. Nanofibers have been demonstrated to increase the differentiation rate of neural stem cells and to improve neurite outgrowth more than microfibers. ${ }^{28}$ However, in 3D culture of immune cells, the nanofiber biomaterial itself may affect cellular activities, such as spontaneous activation/deactivation of DCs. It has been shown that monocyte-derived DCs entangled in multiwalled cylindrical carbon nanotubes undergo a phenotypic and functional shift, displaying a lower immunogenic profile. ${ }^{29}$ More importantly, matrix dimensionality can impact cell morphology and adhesion organization. ${ }^{30,31}$ Morphological changes accompanying DC activation include loss of adhesive structures and cytoskeleton reorganization. BM-DCs grown in 2D culture dish were flat and spread freely in the horizontal plane. LPSstimulated BM-DCs showed increased expression of MHC Class II and F-actin on the cell surface in contact with the 2D culture dish. However, in 3D hybrid NFS, LPS-stimulated BM-DCs showed smaller adhesions, and F-actin expression was not correlated to MHC Class II expression. FAK is known to be activated downstream of integrin-mediated adhesion, which is close contact of adherent cells with the $\mathrm{ECM}$, a dynamic process requiring coordinated rearrangement of the cytoskeleton. ${ }^{31,32}$ LPS-stimulated BM-DCs in 3D hybrid NFS showed activated FAK only at sites of adhesion to nanofibers, but not in elongated dendrites, indicating that the process of dendrite formation may not be affected by that of cellular adhesion in a 3D hybrid scaffold. Thus, DCs cultured in 3D hybrid NFS composed of electrospun PCL fibers are equivalent to those in in vivo conditions.

In coculture of BM-DCs and cancer cells in 3D hybrid NSF, cellular interactions were similar to the naturally occurring cross-talk between these two types of cells, through soluble factors and direct cell-cell contact. In contrast, cell-cell interactions alone, but not paracrine signaling, occurred in coculture of BM-DCs and cancer cells in 2D culture dish. Thus, our system can be used to measure the ability of cancer cells to attract DCs. Microfluidic devices can be used to generate chemoattractant gradients, but the gradients are typically 1D. In comparison, 3D hybrid NFS created 3D gradients that mimic chemoattractant gradients in tissue, allowing DCs to sense the local gradient around mitoxantrone-treated cancer cells. Therefore, 3D hybrid NFS can be used as a coculture system for investigation of cellular cross-talk between cancer and immune cells, including DCs. Reconstruction of tumor tissue using cancer cells and cancer-associated stromal cells may be possible in 3D hybrid NFS, which can also be used as in vitro 3D culture system mimicking in vivo conditions. Therefore, hybrid NFS can be applied to different translational cancer research studies. It is possible to compare mouse xenograft subcutaneous model with a mouse model grafted with cancer cells grown inside the hybrid NFS. Additionally, immunogenic potential of anticancer drugs can be screened by culturing human-derived cancer cells and immune cells, such as peripheral blood DCs, in the 3D hybrid NFS. Finally, ex vivo understanding of the cross-talk of cancer and immune cells in tumor microenvironments will be possible.

\section{Conclusion}

The 3D multicellular culture model for cancer and immune cells developed here can form a more physiologically relevant representation of the cancer microenvironment than currently available 2D systems. A more porous scaffold can be generated by electrospinning nano- and submicron-scale PCL fibers on stainless steel mesh. Cancer cells and BM-DCs deeply infiltrated the 3D hybrid NFS, allowing observation of their interactions. In addition, direct addition of BM-DCs to mitoxantrone-treated cancer cells cultured in 3D hybrid NFS enabled BM-DCs to spread, migrate, and adhere to the cancer cells in a 3D manner. Therefore, in vitro coculture of cancer and immune cells using 3D hybrid NFS mimics the immune microenvironment in cancer tissues, and this culture system can be used to screen anticancer compounds for the ability to elicit antitumor immunity. 


\section{Acknowledgments}

This work was supported by the Pioneer Research Center Program through the National Research Foundation of Korea funded by the Ministry of Science, ICT, and Future Planning (2012-0009583, 2012-0009664, and 2012-0009665).

\section{Disclosure}

The authors report no conflicts of interest in this work.

\section{References}

1. Liu W, Thomopoulos S, Xia Y. Electrospun nanofibers for regenerative medicine. Adv Healthc Mater. 2012;1(1):10-25.

2. Lu T, Li Y, Chen T. Techniques for fabrication and construction of three-dimensional scaffolds for tissue engineering. Int J Nanomedicine. 2013;8(1):337-350.

3. Rnjak-Kovacina J, Weiss AS. Increasing the pore size of electrospun scaffolds. Tissue Eng Part B Rev. 2011;17(5):365-372.

4. Tuzlakoglu K, Bolgen N, Salgado AJ, Gomes ME, Piskin E, Reis RL. Nano- and micro-fiber combined scaffolds: a new architecture for bone tissue engineering. J Mater Sci Mater Med. 2005;16(12):1099-1104.

5. Park SH, Kim TG, Kim HC, Yang DY, Park TG. Development of dual scale scaffolds via direct polymer melt deposition and electrospinning for applications in tissue regeneration. Acta Biomater. 2008;4(5): 1198-1207.

6. Banchereau J, Palucka AK. Dendritic cells as therapeutic vaccines against cancer. Nat Rev Immunol. 2005;5(4):296-306.

7. Tesniere A, Schlemmer F, Boige V, et al. Immunogenic death of colon cancer cells treated with oxaliplatin. Oncogene. 2010;29(4):482-491.

8. Li WJ, Danielson KG, Alexander PG, Tuan RS. Biological response of chondrocytes cultured in three-dimensional nanofibrous poly $(\varepsilon-$ caprolactone) scaffolds. J Biomed Mater Res A. 2003;67(4):1105-1114.

9. Li WJ, Tuli R, Huang X, Laquerriere P, Tuan RS. Multilineage differentiation of human mesenchymal stem cells in a three-dimensional nanofibrous scaffold. Biomaterials. 2005;26(25):5158-5166.

10. Martins A, Alves da Silva ML, Faria S, Marques AP, Reis RL, Neves NM. The influence of patterned nanofiber meshes on human mesenchymal stem cell osteogenesis. Macromol Biosci. 2011:11(7);978-987.

11. Wang Y, Wang G, Chen L, et al. Electrospun nanofiber meshes with tailored architectures and patterns as potential tissue-engineering scaffolds. Biofabrication. 2009;1(1):015001

12. Song MG, Hwang SY, Park JI, Yoon S, Bae HR, Kwak JY. Role of aquaporin 3 in development, subtypes and activation of dendritic cells. Mol Immunol. 2011;49(1-2):28-37.

13. Jin JO, Park HY, Xu Q, et al. Ligand of scavenger receptor class A indirectly induces maturation of human blood dendritic cells via production of tumor necrosis factor- $\alpha$. Blood. 2009;113(23):5839-5847.

14. Calderwood DA. Integrin activation. J Cell Sci. 2004;117(5):657-666.

15. Parsons JT, Martin KH, Slack JK, Taylor JM, Weed SA. Focal adhesion kinase: a regulator of focal adhesion dynamics and cell movement. Oncogene. 2000;19(49):5606-5613.
16. Zitvogel L, Kepp O, Senovilla L, Menger L, Chaput N, Kroemer G Immunogenic tumor cell death for optimal anticancer therapy: the calreticulin exposure pathway. Clin Cancer Res. 2010;16(12):3100-3104.

17. Engels B, Rowley DA, Schreiber H. Targeting stroma to treat cancers. Semin Cancer Biol. 2012;22(1):41-49.

18. Fong EL, Lamhamedi-Cherradi SE, Burdett E, et al. Modeling Ewing sarcoma tumors in vitro with 3D scaffolds. Proc Natl Acad Sci U S A. 2013;110(16):6500-6505.

19. Sell SA, McClure MJ, Garg K, Wolfe PS, Bowlin GL. Electrospinning of collagen/biopolymers for regenerative medicine and cardiovascular tissue engineering. Adv Drug Deliv Rev. 2009;61(12):1007-1019.

20. Fu X, Xu M, Liu J, Qi Y, Li S, Wang H. Regulation of migratory activity of human keratinocytes by topography of multiscale collagen-containing nanofibrous matrices. Biomaterials. 2014;35(5):1496-1506.

21. Tayalia P, Mendonca CR, Baldacchini T, Mooney DJ, Mazur E. 3D cell-migration studies using two-photon engineered polymer scaffolds. Adv Mater. 2008;20(23):4494-4498.

22. Badami AS, Kreke MR, Thompson MS, Riffle JS, Goldstein AS Effect of fiber diameter on spreading, proliferation, and differentiation of osteoblastic cells on electrospun poly(lactic acid) substrates. Biomaterials. 2006;27(4):596-606.

23. Lowery JL, Datta N, Rutledge GC. Effect of fiber diameter, pore size and seeding method on growth of human dermal fibroblasts in electrospun poly(E-caprolactone) fibrous mats. Biomaterials. 2010:31(3); 491-504.

24. Schwartz MA, Chen CS. Cell biology. Deconstructing dimensionality. Science. 2013;339(6118):402-404.

25. Zhong S, Zhang Y, Lim CT. Fabrication of large pores in electrospun nanofibrous scaffolds for cellular infiltration: a review. Tissue Eng Part $B$ Rev. 2012;18(2):77-87.

26. Kidoaki S, Kwon IK, Matsuda T. Mesoscopic spatial designs of nanoand microfiber meshes for tissue-engineering matrix and scaffold based on newly devised multilayering and mixing electrospinning techniques. Biomaterials. 2005;26(1):37-46.

27. Pham QP, Sharma U, Mikos AG. Electrospun poly(E-caprolactone) microfiber and multilayer nanofiber/microfiber scaffolds: characterization of scaffolds and measurement of cellular infiltration. Biomacromolecules. 2006;7(10):2796-2805.

28. Yang F, Murugan R, Wang S, Ramakrishna S. Electrospinning of nano/ micro scale poly(L-lactic acid) aligned fibers and their potential in neural tissue engineering. Biomaterials. 2005;26(25):2603-2610.

29. Aldinucci A, Turco A, Biagioli T, et al. Carbon nanotube scaffolds instruct human dendritic cells: modulating immune responses by contacts at the nanoscale. Nano Lett. 2013;13(12):6098-6105.

30. Cukierman E, Pankov R, Stevens DR, Yamada KM. Taking cellmatrix adhesions to the third dimension. Science. 2001;294(5547) 1708-1712.

31. Huveneers S, Danen EH. Adhesion signaling - crosstalk between integrins, Src and Rho. J Cell Sci. 2009;122(8):1059-1069.

32. Zamir E, Geiger B. Molecular complexity and dynamics of cell-matrix adhesions. J Cell Sci. 2001;114(20):3583-3590.
International Journal of Nanomedicine

\section{Publish your work in this journal}

The International Journal of Nanomedicine is an international, peerreviewed journal focusing on the application of nanotechnology in diagnostics, therapeutics, and drug delivery systems throughou the biomedical field. This journal is indexed on PubMed Central, MedLine, CAS, SciSearch ${ }^{\circledR}$, Current Contents ${ }^{\circledR} /$ Clinical Medicine,

\section{Dovepress}

Journal Citation Reports/Science Edition, EMBase, Scopus and the Elsevier Bibliographic databases. The manuscript management system is completely online and includes a very quick and fair peer-review system, which is all easy to use. Visit http://www.dovepress.com/ testimonials.php to read real quotes from published authors. 